\title{
Comparison of progression of macrovascular diseases after kidney or pancreas and kidney transplantation in diabetic patients with end-stage renal disease
}

\author{
G. Biesenbach ${ }^{1}$, R. Margreiter ${ }^{2}$, A.Königsrainer ${ }^{2}$, C. Bösmüller ${ }^{2}$, O.Janko ${ }^{1}$, P. Brücke ${ }^{3}$, C. Gross ${ }^{3}$ J Zazgornik $^{1}$ \\ ${ }^{1}$ Second Department of Medicine, General Hospital, Linz, Austria \\ ${ }^{2}$ Department of Transplant Surgery, University Hospital, Innsbruck, Austria \\ ${ }^{3}$ First Department of Surgery, General Hospital Linz, Austria
}

\section{Abstract}

Aims/hypothesis. The aim of the study was to examine the effect of pancreas-kidney transplantation on the progression of macrovascular diseases in Type I diabetic patients with end-stage renal disease.

Methods. The progression of cerebrovascular disease, coronary heart disease and peripheral vascular disease in uraemic patients with Type I (insulin-dependent) diabetes mellitus and who had had simultaneous pancreas-kidney transplantation was compared with that of recipients of a kidney transplant alone. Between 1986 and 1998 a total of 11 uraemic diabetic patients received a simultaneous pancreaskidney transplantation and 10 diabetic patients a kidney transplant alone. All transplants functioned for at least 24 months, the mean observation period was $69 \pm 37$ compared with $70 \pm 33$ months in both patient groups. Macroangiopathic diseases were classified in four stages as described earlier.

Results. In the group with simultaneous pancreas-kidney transplantation progression of cerebrovascular and coronary heart disease was observed in four pa- tients $(36 \%)$ and progression of peripheral vascular disease in five subjects (45\%). In the cohort with kidney transplant alone four patients $(40 \%)$ showed progression of cerebrovascular and coronary heart disease and five progression of peripheral vascular disease $(50 \%)$; the difference is not significant. Mean values of $\mathrm{HbA}_{1 \mathrm{c}}(5.8 \pm 0.2$ vs $7.5 \pm 0.6 \%$, $p<0.001)$ and serum triglycerides $(1.2 \pm 0.4$ vs $2.0 \pm 1.0 \mathrm{mmol} / 1, p<0.05$ ) were significantly lower in the patients with pancreas-kidney transplantation than in the patient group with kidney transplant alone. Serum cholesterol concentrations and blood pressures were similar in both cohorts.

Conclusion/interpretation. From our results we concluded that pancreas-kidney transplantation reduces risk factors for the development of macroangiopathy but fails to halt progression of macrovascular diseases similar to Type I diabetic patients with kidney transplant alone. [Diabetologia (2000) 43: 231-234]

Keywords Type I diabetes, pancreas-kidney transplantation, macroangiopathic diseases.
Simultaneous pancreas-kidney transplantation (SPKT) has become the therapy of choice in patients

Received: 10 August 1999 and in revised form: 15 October 1999

Corresponding author: Dr. G. Biesenbach, 2nd Department of Medicine, General Hospital Linz, Krankenhausstrasse 9, A-4020 Linz, Austria

Abbreviations: CVD, Cerebrovascular disease; CHD, coronary heart disease; PVD, peripheral vascular disease; SPKT, simultaneous pancreas-kidney transplantation; KTA, kidney transplant alone. with Type I (insulin-dependent) diabetes mellitus who have end-stage renal disease. During the last decade graft and patient survival have been improved, the 1-year pancreas graft survival rate is currently at $81 \%$ for SPKT [1].

Several studies have shown that diabetic retinopathy and peripheral neuropathy are positively influenced by pancreas transplantation [2,3]. A recent study also showed that pancreas transplantation in non-uraemic patients can reverse lesions of diabetic nephropathy, but this is not seen before 5 years of normoglycaemia [4]. 
There are only little data in the literature concerning the effect of pancreas transplantation on the progression of macroangiopathy. One study compared the progression of peripheral vascular disease of pancreas-kidney recipients with that of patients who received a kidney transplant alone (KTA) [5]. Despite insulin independence, pancreas-kidney recipients had a statistically significantly higher incidence of peripheral vascular complications. In a more recent study the incidence of peripheral vascular complications in recipients of SPKT was similar to that of KTA patients [6].

In our retrospective analysis the progression of cerebrovascular disease (CVD) and peripheral vascular disease (PVD) as well as coronary heart disease (CHD) in patients with SPKT was compared with that of KTA. In addition, risk factors for atherosclerosis were investigated in both groups of patients.

\section{Subjects and methods}

Only Type I diabetic patients with SPKT or KTA whose transplants functioned for at least 2 years were included in this study. Between 1987 and 1996, 22 Type I diabetic patients who were routinely followed at our outpatient care unit, developed end-stage diabetic nephropathy. One patient died 12 months after initiation of haemodialysis. Of the patients nine underwent KTA at the First Department of Surgery in our hospital. In 12 patients SPKT was done at the Department of Transplant Surgery of the University Innsbruck. Segmental pancreas transplantation with systemic drainage and exocrine diversion to the bladder was carried out in all cases. One recipient lost the pancreas graft immediately after transplantation due to venous thrombosis. In all other cases transplants functioned at least 2 years. Therefore, 11 Type I diabetic patients with functioning SPKT (women:men $=7: 4$, mean age $35 \pm 6$ years, diabetes duration $19 \pm 3$ years) and 10 subjects with KTA (women:men $=7: 3$, mean age $41 \pm 8$ years, diabetes duration $21 \pm 4$ years) were evaluated. Renal grafts were considered functioning as long as patients did not require dialysis (glomerular filtration rate $>20 \mathrm{ml} \cdot \mathrm{min}^{-1} \cdot\left(1.73 \mathrm{~m}^{2}\right)^{-1}$ ). Pancreas graft function was defined as near-normal blood glucose without exogenous insulin. Immunosuppression was the same in all patients using initially a triple drug therapy with prednisone, azathioprine and cyclosporin. The mean observation period was $69 \pm 37$ (24-132) months in the patients with SPKT and $70 \pm 33$ (24-138) months in the patients with KTA. All patients were examined within 6 months before transplantation and monthly after transplantion. At each visit to our outpatient care unit serum creatinine, blood urea nitrogen, haemoglobin, nocturnal blood sugar (multichannel autoanalyser, Hitachi, Boehringer, Vienna, Austria), cyclosporin concentration (monospecific immunoassay, Behring Diagnostics, Vienna, Austria) and the blood pressure (mercury sphygmomanometer) were determined. Total cholesterol and triglycerides in serum, as well as fibrinogen (multichannel autoanalyser, Hitachi) and $\mathrm{HbA}_{1 \mathrm{c}}$ (microcolumn chromatography, Bio-Rad, Vienna Austria, normal range: $4.3-5.9 \%$ ) were measured at 2-monthly intervals.

To evaluate the prevalence and progression of macroangiopathic disease Doppler blood flow studies of the carotid arteries as well of the peripheral arteries of the lower legs, and a 12-lead electrocardiogram (ECG) at rest were carried out within 6 months before transplantation and at the end of the observation period (at the end of graft function or in patients with functioning transplants at the end of 1998). The two main Dopplers used were Linear Ultrasound Scanner 7.4 MHz, Ultramark 5 and Ultramark 9 (Advanced Technology Laboratories, Vienna, Austria). A thallium scan was also done before transplantation and coronary angiography when clinically indicated (abnormal ECG or thallium scan) and in all diabetic patients over 40 years of age.

Mean values of the serum lipids, fibrinogen, nocturnal blood sugar, blood pressure and $\mathrm{HbA}_{1 \mathrm{c}}$ were evaluated and the prevalence of cerebrovascular and peripheral vascular diseases and of CHD were compared in both groups of patients before transplantation and at the end of the observation period. Vascular diseases were classified in four stages: CVD: stage I; carotid artery stenosis less than $50 \%$, stage II; stenosis $50 \%$ or more without clincial symptoms, stage III; stenosis more than $80 \%$ or clinical symptoms or both, stage IV: stroke in medical history. CHD: stage I; ischaemic changes in ECG or angina pectoris at physical exercise, stage II; ischaemic changes in ECG or angina pectoris at rest, stage III; angioplasty or bypass surgery, stage IV; history of myocardial infarction. PVD: stage I; mediasclerosis or ankle/brachial pressure index 1.0-0.7, stage II; an$\mathrm{kle} / \mathrm{brachial}$ pressure index less than 0.7 without symptoms, stage III; ankle/brachial pressure less than 0.7 with pain, stage IV; ischaemic ulceration requiring amputation.

Statistical methods. Data are given as means (SD) or prevalences. For the statistical analysis differences between the groups were tested with the chi-squared test and the student's $t$ test for unequal variances. A $p$ value of less than 0.05 was considered significant.

\section{Results}

In the patient group with SPKT one patient (9\%) showed CVD stage I before transplantation, two patients $(18 \%)$ CHD stage I and four patients (36\%) PVD, stage I $(n=3)$ and II $(n=1)$. At the end of the observation period four patients $(36 \%)$ had CVD, stage I $(n=2)$ and stage IV $(n=2)$ as well as CHD, stage I $(n=3)$ and stage IV $(n=1)$. In six patients $(55 \%)$ PVD stage I $(n=3)$ or stage II $(n=1)$ and stage IV $(n=2)$ was observed. In summary, progression of CVD and CHD was seen in four patients $(36 \%)$ and during the same period five subjects (45\%) showed progression of PVD (Fig. 1, left side). In the patient group with KTA two subjects $(20 \%)$ had CVD stage I as well as CHD stage I and four patients $(40 \%)$ showed PVD stage I $(n=3)$ and stage III $(n=1)$ before transplantation. At the end of the observation period five of the patients $(50 \%)$ had CVD stage I $(n=3)$ and stage IV $(n=2)$, four $(40 \%)$ had CHD stage I $(n=1)$ or stage II $(n=1)$ and stage IV $(n=2)$. Progression of CVD and CHD was therefore observed in four patients $(40 \%)$, five patients $(50 \%)$ showed progression in PVD (Fig. 1, right side). The incidence of the macroangiopathic complications was not statistically significantly different between the patients with SPKT and with KTA. 


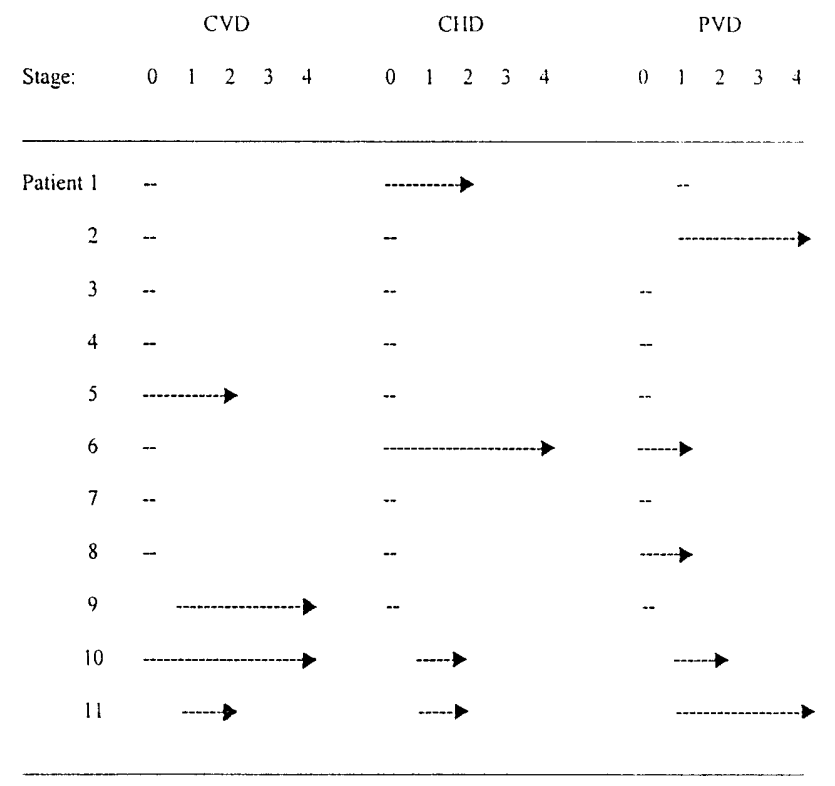

Fig. 1. Prevalences (-) of cerebrovascular disease (CVD), coronary heart disease (CHD) and peripheral vascular disease (PVD) before transplantation and progression $(\rightarrow)$ of macroangiopathic diseases after simultaneous pancreas kidney transplanation (left side) and after kidney transplantation alone (right side)

The mean values of $\mathrm{HbA}_{1 \mathrm{c}}$ were moderately increased $(7.5 \pm 0.6 \%)$ in the patient group with kidney graft alone and normal $(5.8 \pm 0.2 \%)$ in SPKT patients $(p<0.001)$. Mean total cholesterol concentrations were similar in both groups of patients and those of triglyceride were significantly higher in the patients with KTA $(2.0 \pm 1.0$ vs $1.2 \pm 0.4 \mathrm{mmol} / 1, p<0.05)$. Mean fibrinogen concentrations and the mean blood pressure were not significantly different in either cohort. All mean values of metabolic control, serum lipids and blood pressure are summarised in Table 1.

Table 1. Mean values of $\mathrm{HbA}_{1 \mathrm{c}}$, nocturnal blood glucose (BG), serum lipids, fibrinogen and blood pressure (BP) and prevalence of cigarette consumption and angiotensin converting enzyme (ACE)-therapy after transplantation

\begin{tabular}{lll}
\hline & $\begin{array}{l}\text { Simultaneous } \\
\text { pancreas kidney }\end{array}$ & Kidney alone \\
\hline $\mathrm{HbA}_{1 \mathrm{c}}(\%)$ & $5.8 \pm 0.2^{\mathrm{c}}$ & $7.5 \pm 0.6^{\mathrm{c}}$ \\
Fasting BG (mmol/l) & $5.5 \pm 0.6^{\mathrm{c}}$ & $7.4 \pm 1.8^{\mathrm{c}}$ \\
Serum cholesterol $(\mathrm{mmol} / \mathrm{l})$ & $6.3 \pm 1.2^{\mathrm{a}}$ & $6.2 \pm 0.8^{\mathrm{a}}$ \\
Serum triglycerides $(\mathrm{mmol} / \mathrm{l})$ & $1.2 \pm 0.4^{\mathrm{b}}$ & $2.0 \pm 1.0^{\mathrm{b}}$ \\
Fibrinogen $(\mathrm{g} / \mathrm{l})$ & $3.9 \pm 0.9^{\mathrm{a}}$ & $3.6 \pm 0.9^{\mathrm{a}}$ \\
Systolic BP $(\mathrm{mm} \mathrm{Hg})$ & $138 \pm 8^{\mathrm{a}}$ & $142 \pm 8^{\mathrm{a}}$ \\
Diastolic BP $(\mathrm{mm} \mathrm{Hg})$ & $83 \pm 5^{\mathrm{a}}$ & $84 \pm 6^{\mathrm{a}}$ \\
Smoking $(n)$ & $3 / 11^{\mathrm{a}}$ & $2 / 10^{\mathrm{a}}$ \\
ACE-therapy $(n)$ & $1 / 11^{\mathrm{a}}$ & $2 / 10^{\mathrm{a}}$ \\
\hline
\end{tabular}

${ }^{\mathrm{a}} \mathrm{NS},{ }^{\mathrm{b}} p<0.05,{ }^{\mathrm{c}} p<0.001$
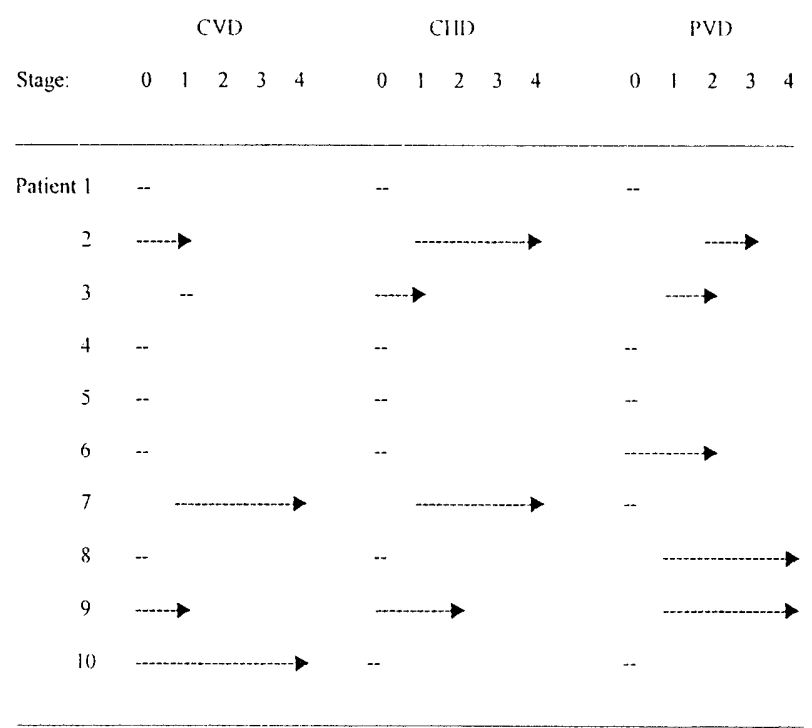

\section{Discussion}

Progression of macroangiopathic disease in patients after pancreas-kidney transplantation is still a matter of debate. Earlier studies investigated only the effect of SPKT on the progression of peripheral vascular disease in Type I diabetic patients.

The results of these studies $[5,6]$ were controversial; the incidence of peripheral vascular complications in recipients of SPKT was either similar or higher in comparison with patients with KTA. In a recent study a substantial reduction in mortality in Type I diabetic patients 10 years after successful SPKT was, however, found [7]. In our study we compared the progression of CVD, CHD and PVD in diabetic patients with SPKT and KTA. The results of our study show that the progression of macroangiopathy in patients with SPKT is similar to that of the diabetic patients with KTA, although control of carbohydrate metabolism was excellent in SPKT patients. The reason for this increased risk for atherosclerosis also in patients with SPKT is not known.

The patients in our study with SPKT had significantly lower concentrations of blood glucose and also triglycerides than did those with KTA. Serum cholesterol concentrations were moderately increased in both cohorts but there were no significant differences between the mean values. Our data confirm a previous study [8] showing similar total cholesterol concentrations in diabetic patients with endstage renal disease and in SKPT and KTA recipients.

Increased fibrinogen concentrations have been described after SPKT as well as KTA [9]. In our study the mean concentrations of fibrinogen were higher in the patients with SPKT but differences were not significant. Mean blood pressure values were similarly increased in both groups of our patients. This might in part be explained by the cyclosporin received by all 
patients. In other studies persisting hypertension as a main risk factor for atherosclerosis was also observed in both patients with SPKT and KTA and was found to be associated with cyclosporin therapy [9]. The prevalence of smokers and patients with angiotensin converting enzyme inhibitor therapy was similar in both groups of our patients.

New immunosuppressive drugs such as tacrolimus, which is known not to impair lipid metabolism, together with portal drainage of the pancreas graft could lead to a more effective prevention of the progression of macrovascular disease in patients with SPKT [10].

The controversial results in the literature concerning the effect of SPKT on progression of macrovascular diseases could be because of the small groups of patients in most of the studies. Moreover, from previous studies, it is known that microangiopathic lesions are affected by pancreas transplantation only after several years [2]. From our patients four with SPKT and three of the recipients of KTA had a follow-up of less than 4 years; this might have some bearing on our negative findings in the SPKT group. Dialysis duration was significantly longer in our patients with KTA than in the patients with SPKT $(6 \pm 5$ vs $24 \pm 17$ months, $p<0.01)$. There was no difference in the smoking history in both groups of transplant recipients.

Our study shows that SKPT does not have the potency to halt progression of macroangiopathy but the numbers of patients were small in both groups. Due to a lower incidence of lethal cardiovascular events long-term survival of patients with SPKT is clearly higher than in diabetic patients with KTA [7]. In our study the 5 -year survival rate was $82 \%$ in the patients with SPKT compared with $70 \%$ in the KTA recipients (NS).

From our findings we conclude that progression of CVD, CAD and PVD is similar in the diabetic patients with SPKT and KTA, although recipients of
KTA have higher blood sugar concentrations and hypertriglyceridaemia. Other atherosclerotic risk factors must be responsible for the similar progression of macroangiopathy.

\section{References}

1. Sutherland DER (1998) Pancreas and pancreas-kidney transplantation. Curr Opin Nephrol Hypertens 7: 317-325

2. Landgraf R (1996) Impact of pancreas transplantation on diabetic secondary complications and quality of life. Diabetologia 39: 1415-1424

3. Königsrainer A, Miller K, Steurer W et al. (1991) Does pancreas transplantation influence the course of diabetic retinopathy? Diabetologia 34[Suppl 1]: 86-88

4. Fioretto P, Steffes MW, Sutherland DER, Goetz FC, Mauer M (1998) Reversal of lesions of diabetic nephropathy after pancreas transplantation. N Engl J Med 339: 69-75

5. Morrissey PE, Shaffer D, Monaco AP, Conway P, Madras PN (1997) Peripheral vascular disease after kidney-pancreas transplantation in diabetic patients with end-stage renal disease. Arch Surg 132: 358-362

6. Knight RJ, Schanzer H, Guy S, Fishbein T, Burrows L, Miller C (1998) Impact of kidney-pancreas transplantation on the progression of peripheral vascular diasease in diabetic patients with end-stage renal disease. Transplant Proc 30: 1947-1949

7. Tyden G, Bolinder J, Solders G, Brattström C, Tibell A, Groth CG (1999) Improved survival in patients with insulin-dependent diabetes mellitus and end-stage diabetic nephropathy 10 years after combined pancreas and kidney transplantation. Transplantation 67: 645-648

8. La Rocca E, Secchi A, Parlavecchia M et al. (1995) Lipoprotein profile after combined kidney-pancreas transplantation in insulin-dependent diabetes mellitus. Transpl Int 8: $190-195$

9. Kasiske BL (1988) Possible causes and consequences of hypertension in stable renal transplant patients. Transplantation 44: 639-643

10. Henley SE, Larsen JL, Mack-Shipman L, Miller SA, Cushing KA, Stratta RJ (1995) Lipids following pancreas transplantation in recipients receiving FK 506. Transplant Proc 27: 2997 